\title{
Formation rates of iron-acceptor pairs in crystalline silicon
}

\author{
Daniel Macdonald and Thomas Roth ${ }^{\mathrm{a})}$ \\ Department of Engineering, Information Sciences and Engineering Institute, The Australian National \\ University, Canberra, ACT 0200, Australia \\ Prakash N. K. Deenapanray \\ Centre for Sustainable Energy Systems, Information Sciences and Engineering Institute, The Australian \\ National University, Canberra, ACT 0200, Australia
}

\begin{abstract}
Karsten Bothe, Peter Pohl, and Jan Schmidt
Institut für Solarenergieforschung Hameln/Emmerthal (ISFH), Am Ohrberg 1, D-31860 Emmerthal, Germany
\end{abstract}

(Received 25 July 2005; accepted 2 September 2005; published online 19 October 2005)

\begin{abstract}
The characteristic association time constant describing the formation of iron-acceptor pairs in crystalline silicon has been measured for samples of various $p$-type dopant concentrations and species $(\mathrm{B}, \mathrm{Ga}$, and $\mathrm{In})$ near room temperature. The results show that the dopant species has no impact on the pairing kinetics, suggesting that the pairing process is entirely limited by iron diffusion. This conclusion was corroborated by measurement of the activation energy of pair formation, which coincides with the migration enthalpy of interstitial iron in silicon. The results also indicate that the pair-formation process occurs approximately twice as fast as predicted by a commonly used expression. (C) 2005 American Institute of Physics. [DOI: 10.1063/1.2102071]
\end{abstract}

\section{INTRODUCTION}

Interstitial iron $\left(\mathrm{Fe}_{i}\right)$ is a common impurity in modern photovoltaic-grade crystalline silicon materials, ${ }^{1}$ and is often a dominant source of carrier recombination. In $p$-type crystalline silicon, the electrostatic attraction between the positively charged $\mathrm{Fe}_{i}$ and negatively charged ionized acceptor atoms causes them to form $\mathrm{Fe}_{i}$-acceptor pairs that are stable at room temperature. Although these pairs dissociate under illumination, and hence do not exist under normal solar cell operating conditions, their presence after pair formation in the dark can be exploited, through carrier lifetime measurements, to obtain useful information about Fe contamination in the starting material, or occurring during device processing. For example, the total interstitial Fe concentration can be calculated from such lifetime measurements before and after pair breaking. This technique was initially developed for $\mathrm{FeB}$ pairs using low-injection surface photovoltage (SPV) measurements, ${ }^{2}$ and has been extended recently to other injection levels ${ }^{3}$ and also to $\mathrm{FeGa}$ and FeIn pairs. ${ }^{4}$

Another useful property of Fe-acceptor pairs is that they allow for unambiguous identification of the presence of interstitial Fe. This can be achieved by measuring the characteristic time constant of Fe-acceptor pair formation after dissociation by either illumination or thermal activation. ${ }^{2}$ Such association times have been measured for $\mathrm{FeB}$ pairs in numerous studies, ${ }^{5-12}$ the most widely used results being those of Zoth and Bergholz, ${ }^{2}$ who determined an expression for the $\mathrm{FeB}$ pair association time constant as a function of boron concentration and temperature based on a model of Reiss et $a l .{ }^{13}$ If the pair-formation process is limited by diffusion of $\mathrm{Fe}_{i}$, as is suggested by measurements of the activation energy

\footnotetext{
${ }^{a)}$ Present address: Fraunhofer Institute for Solar Energy Systems, Heidenhofstr. 2, D-79110 Freiburg, Germany.
}

for pair formation, the same expression should be valid for other $p$-type dopant species also. However, this has not been confirmed experimentally. ${ }^{14}$

The purpose of this work is to determine such association time constants for Ga- and In-doped samples, and to compare them to B-doped samples. Due to the presence of carrier-induced boron-oxygen defects, ${ }^{15}$ there has been a growing interest in $p$-type dopants other than boron for photovoltaic applications. As a result, such information regarding the pairing kinetics of other Fe-acceptor pairs should become of increasing value. In addition, we compare the association times in photovoltaic-grade multicrystalline silicon to both single-crystal float-zone (FZ) and Czochralskigrown $(\mathrm{Cz})$ silicon, in order to reveal any impact of oxygen impurities or crystallographic defects. We also compare our data to literature values for B-doped samples, which vary by approximately a factor of 2 for a given temperature and doping. The weight of the literature data, as well as our own results, suggest that the pair formation is approximately twice as rapid as determined by Zoth and Bergholz. ${ }^{2}$

\section{IRON-ACCEPTOR PAIR FORMATION}

Reiss et al. ${ }^{13}$ considered ion-pairing reactions in semiconductors in some detail. They showed that if the mobile ions are randomly distributed throughout the semiconductor, and are present in much lower concentrations than the fixed dopant ions, then the unpaired fraction of mobile ions will decay exponentially with a characteristic time constant given by

$$
\tau_{\text {assoc }}=\frac{\varepsilon \varepsilon_{0} k T}{4 \pi q^{2} N D},
$$

where $\varepsilon$ is the dielectric constant of the semiconductor, $N$ the ionized dopant concentration, and $D$ the diffusivity of the 


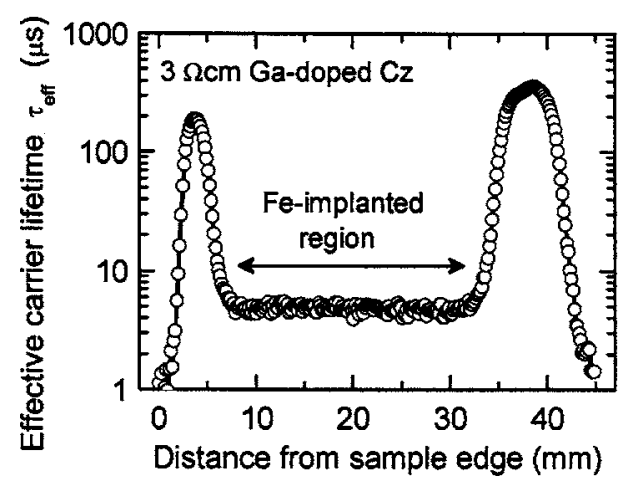

FIG. 1. Cross section through an infrared camera lifetime image of an iron-implanted, annealed, and surface-passivated sample.

oppositely charged mobile ion. For positively charged $\mathrm{Fe}_{i}$ in $p$-type silicon, for which the diffusivity is a simple exponential function of temperature $D=D_{0} \exp \left(-H_{M} / k T\right),{ }^{16-18}$ and assuming the formation process is diffusion limited, this can be written as

$$
\tau_{\text {assoc }}=\frac{A T}{N_{A}} \exp \left(\frac{H_{M}}{k T}\right) \quad \text { with } A=\frac{\varepsilon \varepsilon_{0} k}{4 \pi q^{2} D_{0}} .
$$

Here $N_{A}$ is the $p$-type dopant concentration and $H_{M}$ is the migration enthalpy of $\mathrm{Fe}_{i}^{+}$in silicon. Thus measurement of $\tau_{\text {assoc }}$ at a given temperature can yield values for $D_{0}$, while temperature-dependent measurements can be used to extract $H_{M}$. Zoth and Bergholz ${ }^{2}$ used $D_{0}$ and $H_{M}(0.68 \mathrm{eV})$ values from Weber $^{17}$ to calculate a prefactor $A$ of 4.3 $\times 10^{5} \mathrm{~s} \mathrm{~cm}^{-3} \mathrm{~K}^{-1}$, which fitted their measurements on $\mathrm{FeB}$ pairs well.

\section{EXPERIMENTAL DETAILS}

The FZ and $\mathrm{Cz}$ samples of various resistivities and p-type dopant species (B, Ga, and $\mathrm{In}$ ) used in this study were initially etched and cleaned prior to Fe contamination. The samples were then implanted with $\mathrm{Fe}^{56}$ to a dose of 1 $\times 10^{11} \mathrm{~cm}^{-2}$ on one surface with an energy of $70 \mathrm{keV}$, using a silicon aperture $\sim 30 \times 30 \mathrm{~mm}^{2}$ in size. After another cleaning sequence, the samples were annealed at $900{ }^{\circ} \mathrm{C}$ in nitrogen for $1 \mathrm{~h}$, distributing the Fe uniformly throughout the sample thickness. Note that the resulting Fe concentrations are below the solid solubility limit at $900{ }^{\circ} \mathrm{C},{ }^{18}$ hence precipitation is avoided. Also note that the low energy and dose used do not result in any residual implantation damage after annealing, ${ }^{19}$ which could otherwise reduce the carrier lifetime. After further cleaning and etching, surface passivation was achieved by plasma-enhanced chemical-vapor-deposited (PECVD) silicon nitride films in order to facilitate sensitive measurement of the bulk lifetime.

The lateral uniformity of the Fe concentration in the implanted samples after annealing and passivation was confirmed with an infrared camera lifetime mapping technique. ${ }^{20}$ Figure 1 shows a line scan taken through such a map of a $3 \Omega \mathrm{cm}$ Ga-doped $\mathrm{Cz}$ sample which was $45 \times 45 \mathrm{~mm}^{2}$ in size, using light of wavelength $880 \mathrm{~nm}$ and a photon flux of $1.5 \times 10^{17} \mathrm{~cm}^{-2} \mathrm{~s}^{-1}$. The Fe-implanted region in the center is clearly visible, and exhibits excellent uniformity. The multi-

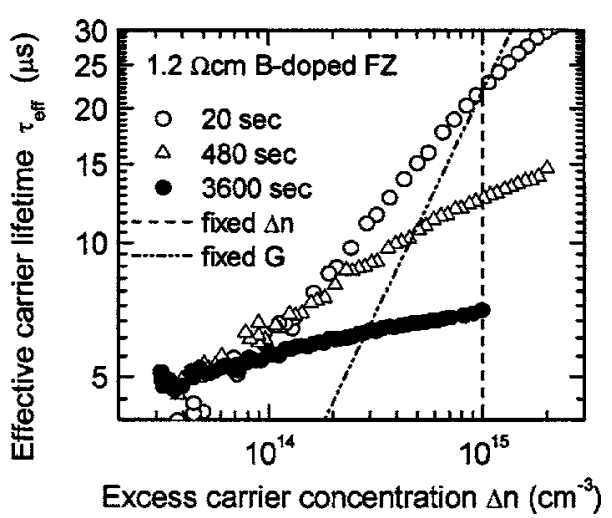

FIG. 2. Effective carrier lifetime as a function of excess carrier density at various times after breaking FeB pairs optically. The dashed lines indicate data extraction at a fixed excess carrier density of $\Delta n=1 \times 10^{15} \mathrm{~cm}^{-3}$, or a fixed generation rate equivalent to 6 suns.

crystalline samples measured in this study were not implanted, but contained varying amounts of natural "grownin" Fe. Although this means that the lateral distribution of $\mathrm{Fe}$ across these wafers may vary considerably, the results nevertheless agree very well with the single-crystal samples.

To determine the association time constants of Feacceptor pairs, effective carrier lifetimes were measured using the quasi-steady-state photoconductance (QSSPC) technique, ${ }^{21}$ which averages the conductance over an area of $\sim 3 \mathrm{~cm}^{2}$, within the implanted region. Lifetime measurements were taken after the samples had rested in the dark for at least one week $\left(\tau_{\text {dark }}\right)$, and at various times after breaking the Fe-acceptor pairs with $2 \mathrm{~min}$ of $200 \mathrm{~mW} / \mathrm{cm}^{2}$ white light illumination. As the pairs reform, the lifetime decreases due to the stronger recombination activity of $\mathrm{Fe}$-acceptor pairs at excess carrier densities above $\sim 10^{14} \mathrm{~cm}^{-3}$. 3,4 Examples of such measurements are given in Fig. 2 for the case of a boron-doped FZ sample.

The relative interstitial Fe concentration during the repairing process is simply equal to $1 / \tau_{\text {dark }}-1 / \tau$, where the lifetimes are measured at a fixed excess carrier density $\Delta n$, chosen as $1 \times 10^{15} \mathrm{~cm}^{-3}$ in this work. This approach is quite robust, because subtracting recombination rates before and after illumination subtracts out the impacts of other recombination channels that are not altered by the illumination process, such as Auger and surface recombination. Hence their only effect can be to reduce the sensitivity of the Fe measurement if they become dominant, although in principle they cannot alter its magnitude.

The measured change in the interstitial Fe concentrations after pair breaking is shown in Fig. 3 for some samples. The straight lines represent single exponential decays, which describe the pairing reaction very well, as observed previously. ${ }^{2,6,8}$ The fits yield the characteristic association time $\tau_{\text {assoc. }}$. The sample temperature during these measurements was held at $31 \pm 3{ }^{\circ} \mathrm{C}$ on the QSSPC stage, as monitored by a thermocouple. The slightly elevated temperature compared to room temperature is due to a built-in heating element in the QSSPC system to reduce temperature fluctuations, and the small heating effect of the eddy currents induced in the sample by the detection coil. Note that in general, the measured time constant actually reflects a 


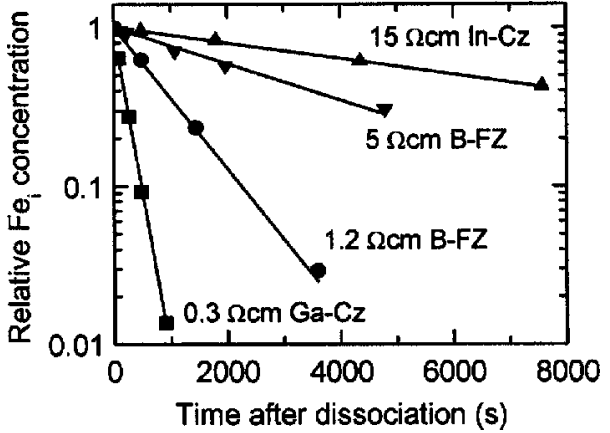

FIG. 3. Reduction in the relative interstitial Fe concentration due to repairing after dissociation for various samples. The straight lines represent exponential decays fitted to extract the association time constants.

combination of the thermally activated association and dissociation rates. ${ }^{18}$ However, at the relatively low temperatures used here, the association rate dominates the measurements in the absence of illumination. Hence we may interpret the characteristic times of Fig. 3 as association time constants. The low temperatures also ensure that Fe precipitation and incomplete pairing in thermal equilibrium do not affect the measurements. ${ }^{18}$

However, some precautions do need to be taken. Since the lifetime measurement process requires illumination with a flash, it can potentially rebreak some Fe-acceptor pairs, resulting in an apparently increased association time. To avoid this, only a small number of well-spaced measurements (several minutes at least) were made for each sample, and filters were used to only generate the intensity required to reach $\Delta n=1 \times 10^{15} \mathrm{~cm}^{-3}$ and not more. Repeated measurements with twice as frequent flashes revealed no change in the measured association time constant, indicating that little rebreaking occurred. Another potential pitfall is that the QSSPC technique estimates the average carrier density via the photoconductance divided by the sample thickness. In cases where the measured lifetime results in a minoritycarrier diffusion length $L_{D}$ less than half the sample thickness, this assumption becomes increasingly inaccurate. In such cases, we replaced the sample thickness with $2 L_{D}$ when calculating the average excess carrier density $\Delta n .^{22}$ This was necessary for some of the more heavily doped samples in which the carrier lifetime at $\Delta n=1 \times 10^{15} \mathrm{~cm}^{-3}$ was short.

We also found that illumination times significantly longer than 2 min were necessary for the most lightly doped samples in order to achieve a pair-formation rate that was independent of illumination time. This is apparently due to the much larger spacing between dopant atoms, requiring a longer period of illumination-enhanced Fe diffusion to yield a truly randomized $\mathrm{Fe}$ distribution. ${ }^{6}$ Indeed for samples with resistivities above $10 \Omega \mathrm{cm}$, thermal pair breaking is preferable due to the faster Fe diffusion, and also the slower repairing, meaning less rapid quenching is required than for more heavily doped samples. For such lightly doped samples we used annealing at $200{ }^{\circ} \mathrm{C}$ for $10 \mathrm{~min}$, followed by quenching in water. Note that for accurate measurement of the association time constant, complete dissociation is not necessary, although those pairs which are dissociated should be fully randomized.

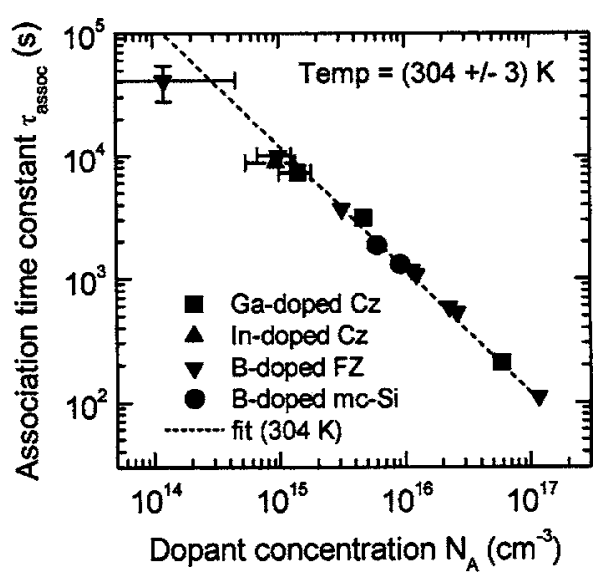

FIG. 4. Association time constants vs doping concentration measured at $(304 \pm 3) \mathrm{K}$ for samples of various growth techniques and $p$-type dopant species. The dashed line is a fit of Eq. (2) with $A=5.0 \times 10^{5} \mathrm{~s} \mathrm{~cm}^{-3} \mathrm{~K}^{-1}$ and $H_{M}=0.66 \mathrm{eV}$.

With these precautions in place, the QSSPC technique is very well suited to such association time measurements, since it explores a wide range of excess carrier densities $\Delta n$, meaning that the carrier lifetime can easily be extracted at a given value of $\Delta n$. Other mid- or high-injection techniques, such as microwave-detected photoconductance decay, or techniques based on free-carrier absorption or emission, generally use a fixed generation rate $G$ rather than a fixed excess carrier density. This is illustrated in Fig. 2, where a line representing a fixed generation rate equivalent to 6 suns is shown. Due to the strong injection dependence of the carrier lifetime in $p$-type $\mathrm{Si}$ containing $\mathrm{Fe}_{i}$, using such a fixed generation rate leads to a systematic underestimation of the association time constant. In this case the error would only be around $25 \%$, but it can be significantly higher depending on the generation rate used. Note that SPV measurements do not suffer from this problem, since they operate in true lowinjection conditions, for which the carrier lifetimes are independent of both the excess carrier density and the generation rate.

\section{RESULTS AND DISCUSSION}

The characteristic association times measured in this work on a large range of samples are shown in Fig. 4 as a function of dopant concentration at a temperature of $(304 \pm 3)$ K. Firstly, note that the association time decreases linearly with doping, as expected from Eq. (2), which has been fitted to our data (using $H_{M}=0.66 \mathrm{eV}$ and $A=5.0$ $\times 10^{5} \mathrm{~s} \mathrm{~cm}^{-3} \mathrm{~K}^{-1}$ as discussed below), and is represented by the dashed line. Secondly, it is clear that the association time is independent of the crystal-growth technique. This suggests that any trapping of $\mathrm{Fe}_{i}$ at defects such as $\mathrm{O}_{i}$ in $\mathrm{Cz}$ material, or crystal defects in multicrystalline silicon, does not significantly retard the formation of Fe-acceptor pairs, despite the fact that these potential "traps" are often much more numerous than the dopant atoms themselves. Thirdly, there is no noticeable effect of dopant species on the Fe-acceptor association time. This implies that the formation process is entirely limited by the diffusion of $\mathrm{Fe}_{i}$, and is not significantly accelerated by Coulombic attraction between the positively 


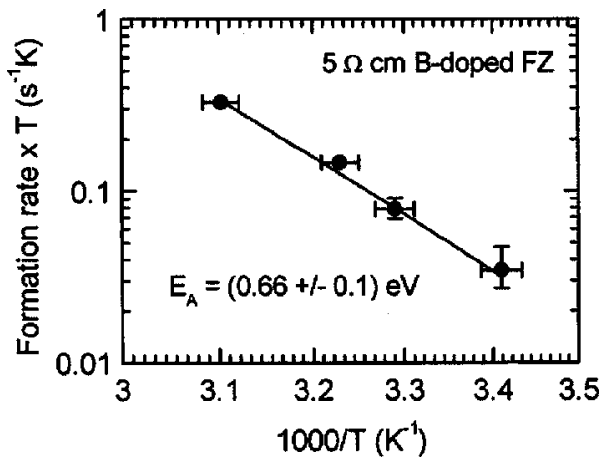

FIG. 5. Arrhenius plot of the pair-formation rate for a B-doped sample, giving an activation energy of pair formation of $E_{A}=(0.66 \pm 0.1) \mathrm{eV}$.

charged $\mathrm{Fe}_{i}$ and the negatively charged dopant atoms. Although such an attraction must exist, its effective range is of the order of $4-5 \mathrm{~nm},{ }^{10,18}$ significantly less than the dopant separation even in the most heavily doped samples used here (around $22 \mathrm{~nm}$ for $N_{A}=10^{17} \mathrm{~cm}^{-3}$ ). However, for doping concentrations higher than approximately $5 \times 10^{18} \mathrm{~cm}^{-3}$, the range of Coulombic attraction becomes comparable to or exceeds the dopant separation. In such cases, which would include Fe gettered to $p$-type diffused regions of devices, pair formation could be enhanced to such an extent that any $\mathrm{Fe}_{i}$ may effectively always exist in the paired state.

Further evidence that the formation process is limited by $\mathrm{Fe}_{i}$ diffusion comes from measurement of the activation energy of pair formation. The pair-formation rate $R_{\text {form }}$ $=1 / \tau_{\text {assoc }}$ was measured at different temperatures for one of the B-doped FZ samples, shown in Fig. 5, yielding an activation energy of $(0.66 \pm 0.1) \mathrm{eV}$. This is very close to the migration enthalpy of $\mathrm{Fe}_{i}$ in crystalline silicon of $0.67-0.68 \mathrm{eV},{ }^{17,18}$ as expected if the pair-formation process is entirely controlled by $\mathrm{Fe}_{i}$ diffusion. The value is in good agreement with measurements by Lagowski et al. ${ }^{8}$ who used the SPV technique in low injection, and Obermeier and Huber $^{11}$ using generation lifetime measurements in metaloxide-semiconductor (MOS) structures (which are therefore also low-injection conditions). However, it is significantly higher than the results of Kaniava et al., ${ }^{9}$ possibly because of their use of a fixed generation rate technique at midinjection, which does not ensure equal excess carrier concentrations for each measurement, or because of pair rebreaking during measurement.

Next, Fig. 6 shows a comparison of our measured association times with some literature values. The solid line represents Eq. (2) with the parameters used by Zoth and Bergholz $\left(H_{M}=0.68 \mathrm{eV}\right.$ and $\left.A=4.3 \times 10^{5} \mathrm{~s} \mathrm{~cm}^{-3} \mathrm{~K}^{-1}\right)$. Note that there is a difference of approximately a factor of 2 between their fit and our own. Figure 6 also shows other data collected from the literature. Only work which specified the sample temperature explicitly is shown, meaning those studies which simply stated "room-temperature" conditions have been excluded. This is an important aspect, since only an 8 -degree increase in temperature would bring Zoth and Bergholz's fit into line with our own data. In all cases shown, the formation rates were measured at $(304 \pm 3) \mathrm{K}$, or extracted at $304 \mathrm{~K}$ from temperature-dependent fits. Furthermore, we

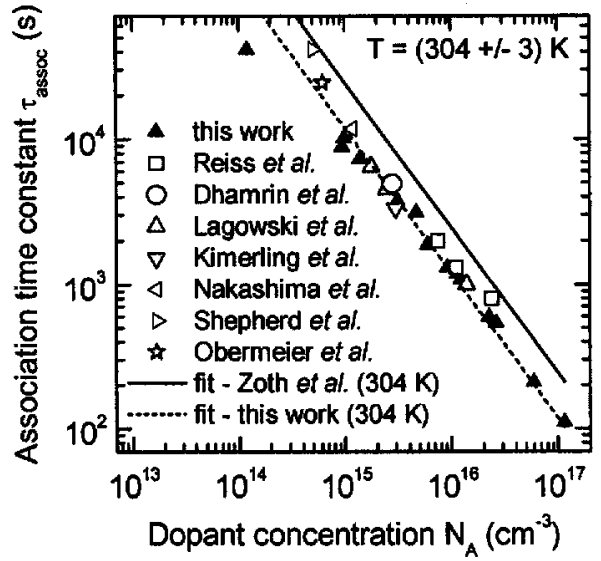

FIG. 6. Association time constants vs doping concentration measured at $(304 \pm 3) \mathrm{K}$ from this work and from the literature. The solid line represents Eq. (2) with the parameters of Zoth and Bergholz, and the dashed line with the parameters determined in this work.

have only shown data for which the methodology used would be expected to generate reliable results, hence excluding studies based on mid- or high-injection techniques that did not account for changing excess carrier densities. The techniques used include $\mathrm{SPV}^{8}$ deep-level transient spectroscopy, ${ }^{6,7}$ generation lifetime measurements, ${ }^{11}$ dark conductivity, ${ }^{5}$ and internal quantum efficiency measurements of solar cells. ${ }^{10}$ All of these techniques ensure low-injection conditions prevail, at least in the probed region of the samples. In all cases B-doped samples were used, except for the result of Dhamrin et al. ${ }^{23}$ on Ga-doped multicrystalline silicon, who used the same approach as in this work, extracting carrier lifetimes at a fixed $\Delta n$ value from QSSPC data. Although they did not specify the sample temperature, it seems reasonable to assume that it would be similar to our own QSSPC apparatus (304 K). Although there is some scatter in the data, the majority of the literature values support our own data set and fit quite well. The fit implies an $\mathrm{Fe}_{i}$ diffusivity of $1.4 \times 10^{-14} \mathrm{~cm}^{2} / \mathrm{s}$ at $304 \mathrm{~K}$, twice as high as the review of Weber, ${ }^{17}$ and at the upper end of the range specified in the review of Istratov et al. ${ }^{18}$ (from 5 $\times 10^{-15}$ to $\left.1.4 \times 10^{-14} \mathrm{~cm}^{2} / \mathrm{s}\right)$.

Finally, in fitting Eq. (2) to our data, one might at first assume the same activation energy of $0.68 \mathrm{eV}$ as used by Zoth and Bergholz, resulting in a reduced value of the prefactor $A=2.2 \times 10^{5} \mathrm{~s} \mathrm{~cm}^{-3} \mathrm{~K}^{-1}$. This results in a fit which is indistinguishable from the dashed lines in Figs. 4 and 6, and therefore could be considered a reasonable parameterization of our results. However, it also causes a significant overestimation of the diffusivity at much higher temperatures $\left(>700{ }^{\circ} \mathrm{C}\right) .{ }^{17,18}$ On the other hand, using an activation energy of $0.66 \mathrm{eV}$, as determined from our own temperaturedependent measurements, yields a prefactor of $A=5.0$ $\times 10^{5} \mathrm{~s} \mathrm{~cm}^{-3} \mathrm{~K}^{-1}$ when fitted to our data, and gives a much better agreement with high-temperature $\mathrm{Fe}_{i}$ diffusivity data. This in turn implies a diffusivity prefactor of $D_{0}=1.1$ $\times 10^{-3} \mathrm{~cm}^{2} \mathrm{~s}^{-1}$, in reasonable agreement with typical literature values. ${ }^{16-18}$ In fact, these values of $H_{M}$ and $D_{0}$ are identical to those determined by Nakashima et al. ${ }^{24}$ from $\mathrm{Fe}_{i}$ diffusivity measurements across a large temperature range 
from 0 to $1000{ }^{\circ} \mathrm{C}$. Hence we arrive at the following expression, as used in Figs. 4 and 6, for the association time constant of $\mathrm{Fe}_{i}$-acceptor pairs:

$$
\tau_{\text {assoc }}=5.0 \times 10^{5} \frac{T}{N_{A}} \exp \left(\frac{0.66}{k T}\right) .
$$

\section{CONCLUSIONS}

The association time constant for Fe-acceptor pairs was verified to be independent of dopant species, consistent with the formation process being diffusion limited. This was also supported by measurement of the activation energy of pair formation, which was very close to the known migration enthalpy of interstitial Fe. The results suggest a somewhat higher value for the diffusivity of $\mathrm{Fe}_{i}$ near room temperature than is commonly used, a conclusion corroborated by a survey of similar studies in the literature. Assuming an activation energy of $0.68 \mathrm{eV}$, this implies a factor of 2 reduction (from $4.3 \times 10^{5}$ to $2.2 \times 10^{5} \mathrm{~s} \mathrm{~cm}^{-3} \mathrm{~K}^{-1}$ ) in the prefactor of the expression commonly used for determining the association time constant for $\mathrm{FeB}$ pairs as a function of doping and temperature. However, an activation energy of $0.66 \mathrm{eV}$ and a prefactor of $A=5.0 \times 10^{5} \mathrm{~s} \mathrm{~cm}^{-3} \mathrm{~K}^{-1}$ were found to give a better fit to high-temperature diffusion data, while still reproducing the room-temperature data very well. This expression was found to be valid for multicrystalline silicon also, meaning it can reasonably be applied to defected photovoltaicgrade silicon materials.

\section{ACKNOWLEDGMENTS}

This work has been supported by the Australian Research Council and the State of Lower Saxony. The authors are grateful to Prof. R. Elliman and C. Jagadish of the Research School of Physical Sciences and Engineering, ANU, for access to the ion implanter and PECVD reactor, and to Dr. J. Vedde of Topsil, Denmark for supplying some of the silicon wafers. One of the authors (J.S.) acknowledges the support of the Alexander von Humboldt Foundation.

${ }^{1}$ L. Jastrzebski, W. Henley, D. Schielein, and J. Lagowski, J. Electrochem. Soc. 142, 3869 (1995)

${ }^{2}$ G. Zoth and W. Bergholz, J. Appl. Phys. 67, 6764 (1990).

${ }^{3}$ D. Macdonald, L. J. Geerligs, and A. Azzizi, J. Appl. Phys. 95, 1021 (2004).

${ }^{4}$ J. Schmidt and D. Macdonald, J. Appl. Phys. 97, 113712 (2005).

${ }^{5}$ W. H. Shepherd and J. A. Turner, J. Phys. Chem. Solids 23, 1697 (1962).

${ }^{6}$ L. C. Kimerling and J. L. Benton, Physica B \& C 116B, 297 (1983).

${ }^{7}$ H. Nakashima, T. Isobe, Y. Yamamoto, and K. Hashimoto, Jpn. J. Appl. Phys., Part 1 27, 1542 (1988).

${ }^{8}$ J. Lagowski, P. Edelman, A. M. Kontkiewicz, O. Milic, W. Henley, M. Dexter, L. Jastrzebski, and A. M. Hoff, Appl. Phys. Lett. 63, 3043 (1993).

${ }^{9}$ A. Kaniava, E. Gaubas, J. Vaitkus, J. Vanhellemont, and A. L. P. Rotondaro, Mater. Sci. Technol. 11, 670 (1995).

${ }^{10}$ J. H. Reiss, R. R. King, and K. W. Mitchell, Appl. Phys. Lett. 68, 3302 (1996).

${ }^{11}$ G. Obermeier and D. Huber, J. Appl. Phys. 81, 7345 (1997).

${ }^{12}$ A. Cacciato, S. Vleeshouwers, and S. Evseev, J. Electrochem. Soc. 145, 701 (1998).

${ }^{13}$ H. Reiss, C. S. Fuller, and F. J. Morin, Bell Syst. Tech. J. 35, 535 (1956).

${ }^{14}$ S. Zhao, L. V. C. Assali, J. F. Justo, G. H. Gilmer, and L. C. Kimerling, J. Appl. Phys. 90, 2744 (2001).

${ }^{15}$ K. Bothe, R. Sinton, and J. Schmidt, Prog. Photovoltaics 13, 287 (2005).

${ }^{16}$ K. Graff, Metal Impurities in Silicon-Device Fabrication, Springer Series in Material Science, Vol. 2 (Springer-Verlag, Berlin, 2000).

${ }^{17}$ E. R. Weber, Appl. Phys. A: Solids Surf. 30, 1 (1983).

${ }^{18}$ A. A. Istratov, H. Hieslmair, and E. R. Weber, Appl. Phys. A: Mater. Sci. Process. 69, 13 (1999).

${ }^{19}$ D. Macdonald, P. N. K. Deenapanray, and S. Diez, J. Appl. Phys. 96, 3687 (2004).

${ }^{20}$ M. Bail, J. Kentsch, R. Brendel, and M. Schulz, in Proceedings 28th IEEE Photovoltaic Specialists Conference, Anchorage, Alaska, 15-22 September 2000 (IEEE, New York, 2000), p. 99.

${ }^{21}$ R. A. Sinton and A. Cuevas, Appl. Phys. Lett. 69, 2510 (1996).

${ }^{22} \mathrm{~S}$. Bowden and R. A. Sinton, in 14th Workshop on Crystalline Silicon Solar Cells and Modules, Winter Park, CO, 8-11 August 2004 (NREL, Golden, CO, 2004).

${ }^{23}$ M. Dhamrin, K. Kamisako, and T. Saitoh, in Proceedings 20th European Photovoltaic Solar Energy Conference, Barcelona, Spain, 6-10 June 2005 (unpublished).

${ }^{24}$ H. Nakashima, T. Sadoh, H. Kitagawa, and K. Hashimoto, Mater. Sci. Forum 143-147, 761 (1994). 